\title{
Stefan Zweig: Ein Mann von Gestern? Ein Interview mit Klemens Renoldner
}

\author{
[Stefan Zweig: A man of yesterday? An Interview with Klemens Renoldner] \\ http://dx.doi.org/10.1590/1982-883718267214235
}

von Ruth Bohunovsky

\begin{abstract}
The curator of the exhibition We need an entirely different courage! Stefan Zweig farewell to Europe, Klemens Renoldner, discusses in this interview the relation between texts and contexts with respect to the work of the Austrian writer Stefan Zweig (1881-1942). He brings out different contexts of reception and different interpretative approaches, that can put traditional stereotypes about the life and work of the author into new perspectives. The conversation also deals with the cooperation between the Stefan Zweig Centre in Salzburg and the Casa Stefan Zweig in Petrópolis (Rio de Janeiro) and mentions joint projects that have already been realized, as well as future projects.
\end{abstract}

Keywords: Stefan Zweig; exile; Brazil

Zusammenfassung: Der Kurator der Ausstellung Wir brauchen einen ganz anderen Mut! Stefan Zweig - Abschied von Europa, Klemens Renoldner, spricht im Interview über Text-KontextBeziehungen in Hinblick auf das Werk des österreichischen Schriftstellers Stefan Zweig (18811942). Dabei kommen u.a. unterschiedliche Rezeptionskontexte und Interpretationsansätze ans Licht, die dazu beitragen, traditionelle Klischees rund um das Leben und das Werk des Autors zu relativieren. Außerdem wird auf die Zusammenarbeit des Stefan Zweig Centres in Salzburg und der Casa Stefan Zweig in Petrópolis (Rio de Janeiro) hingewiesen und abgeschlossene, sowie zukünftige Projekte vorgestellt.

Stichwörter: Stefan Zweig; Exil; Brasilien

Resumo: Klemens Renoldner, curador da exposição Precisamos de uma coragem bem diferente! - Stefan Zweig - despedida da Europa, aborda, em entrevista, as relações entre entre textos e contextos no âmbito da obra do escritor austríaco Stefan Zweig (1881-1942). Com isso, traz à tona diferentes contextos de recepção e vertentes interpretativas da obra zweigiana, contribuindo assim à relativização de tradicionais clichês em torno da vida e da obra do autor. Além disso, a conversa trata da cooperação entre o Stefan Zweig Centre, em Salzburgo, e a Casa Stefan Zweig, em Petrópolis (Rio de Janeiro), e apresenta projetos realizados e futuros.

Palavras-chave: Stefan Zweig; exílio; Brasil

\footnotetext{
${ }^{1}$ Universidade Federal do Paraná, Setor de Ciências Humanas (SCH), Rua General Carneiro, 460, Edifício D. Pedro I, 80.060-150, Curitiba, PR, Brasil. Email: ruth.bohunovsky@uol.com.br
} 


\section{Einleitung}

In den 1920er und 1930er Jahren war er einer der weltweit meistübersetzten Autoren; in Frankreich gilt er nach wie vor als der meistgelesene deutschsprachige Autor; in der Germanistik Deutschlands und Österreichs wird er als “Autor sechster Güte” gehandelt, in einer kürzlich erschienen Fernsehdokumentation des Österreichischen Rundfunks wurde er wiederum (neben Leopoldine von Habsburg) als einer der beiden "prominentesten österreichischen Exilanten in Brasilien" bezeichnet und ein Buch über sein Sexualleben erregt derzeit die Gemüter der Feuilletone Deutschlands und Österreichs. In der englischsprachigen Welt war er lange in Vergessenheit geraten, bis er durch den Film The Grand Budapest Hotel wieder ins Gespräch und in die Publikationslisten kam. In Brasilien wurde nur ein Teil seiner Werke übersetzt, aber 2014 kam sowohl seine Autobiographie in einer Neuübersetzung, als auch sein letztes Telefonbüchlein auf den Markt; seit 2013 arbeitet der Zahar-Verlag an Neuauflagen ausgewähler Werke. Seit 2014 ist in diversen europäischen Städten eine große Ausstellung über sein Leben und Werk im Exil zu sehen, die - so wollen wir hoffen auch nach Brasilien kommen soll. Die Rede ist von Stefan Zweig ${ }^{2}$, dessen Werk und Leben gleichermaßen für seinen anhaltenden Bekanntheitsgrad verantwortlich sind. Im folgenden Interview spricht der Kurator der erwähnten Ausstellung und Direktor des Stefan Zweig Centres in Salzburg, Klemens Renoldner, über den Autor, die neue Ausstellung, die Rezeption und Stellung Zweigs im internationalen Kontext, sowie die Zusammenarbeit des Zweig Centres mit der Casa Stefan Zweig - eine Gedenkstätte für Zweig und andere Exilautoren in seinem letzten Wohnhaus in Petrópolis, Rio de Janeiro.

Die Ausstellung Wir brauchen einen ganz anderen Mut! Stefan Zweig - Abschied von Europa wurde im April 2014 im Wiener Theatermuseum eröffnet, wo sie bis Jänner 2015 zu sehen war. Danach wurde sie im Literaturhaus in München und im Südtiroler

2 Seit 2013 sind im Zahar-Verlag folgende Neuauflagen ausgewähler Werke von Stefan Zweig erschienen, jeweils mit Kommentaren von Alberto Dines: $O$ mundo insone e outros ensaios (2013, übersetzt von Kristina Michahelles); Maria Antonieta (2013, übersetzt von Irene Aron); Autobiografia: o mundo de ontem (2014, übersetzt von Kristina Michahelles); Três novelas femininas - Medo, Carta de uma desconhecida, 24 horas na vida de uma mulher (2014; übersetzt von Adriana Lisboa, Raquel AbiSâmara); Joseph Fouché - retrato de um homem político (2015, übersetzt von Kristina Michahelles), Novelas insólitas - Segredo ardente, Confusão de sentimentos, A coleção invisível, Júpiter, Foi ele?, Xadrez - uma novela (2015, übersetzt von Maria Aparecida Barbosa, Kristina Michahelles e Murilo Jardelino). 2016 soll Cura pelo espírito erscheinen, in einer Übersetzung von Kristina Michahelles. 
Kulturinstitut in Bozen gezeigt und ab 24. November 2015 wird sie für mehrere Monate in der Deutschen Nationalbibliothek in Frankfurt am Main zu besuchen sein. Sie setzt sich mit Leben und Werk des Autors aus der Perspektive des Exils auseinander. Zweigs letzte Exil-Station war Brasilien, wo er sich, gemeinsam mit seiner zweiten Frau Lotte, 1942 in Petrópolis das Leben nahm. Er war damals 60 Jahre alt — hinter ihm lagen eine international erfolgreiche Schriftstellerkarriere und ein im europäischen intellektuellen Kontext äußerst vernetztes Leben, aber auch das Ende seiner "Welt von gestern" durch den Ersten Weltkrieg, die öffentliche Verbrennung seiner Werke durch die Nationalsozialisten und die Zerschlagung seines geistigen Umfelds, die bereits vor dem "Anschluss" Österreichs ans Deutsche Reich und dem Beginn des Zweiten Weltkriegs eingesetzt hatte.

Stefan Zweig war seit Februar 1934 im Exil (er wohnte sechseinhalb Jahre in Großbritannien, danach an verschiedenen Orten in Brasilien, und zwischen Jänner 1941 bis Februar 1942 in Petrópolis). Schon 1933 hatte er in einem Brief an den belgischen Maler Frans Masereel angemerkt, dass er "die stärkste Abneigung [habe], Emigrant zu werden", denn er sei sich bewußt, dass "alles Emigrantentum gefährlich" sei, da es "die Zurückgebliebenen zu Geiseln" mache (vgl. RENOLDNER 2014: 7). Zweig wusste jedoch auch, dass gerade jene Werke, die im Exil entstanden sind, eine hervorragende Bedeutung für ihre jeweiligen Nationalliteraturen haben können (ebd.). Und dies gilt ohne Zweifel für Zweigs Exilwerke, vor allem die Schachnovelle und seine Lebenserinnerungen Die Welt von Gestern - die beide innerhalb seines Gesamtwerks eine hervorragende Stellung einnehmen und im Rahmen der erwähnten Ausstellung besonders gewürdigt werden. Weitere wichtige Werke seiner Exilzeit sind unter anderem: Triumph und Tragik des Erasmus von Rotterdam (1936), Castellio gegen Calvin, oder: Ein Gewissen gegen die Gewalt (1936), die biographischen Arbeiten Maria Stuart (1935), Magellan (1938) und Amerigo Vespucci (1942), der Roman Ungeduld des Herzens (1939), sowie Brasilien. Ein Land der Zukunft (1941).

Die Ausstellung stellt aber nicht nur die Exil-Werks Zweigs in den Mittelpunkt, auch ihre räumliche Gestaltung steht unter dem Zeichen des Exils. Primärer Gestaltungsbaustein ist das mondäne Wiener Hotel Métropole, das von der Gestapo in ein Folterzentrum umfunktioniert wurde - und als Handlungsschauplatz in die Schachnovelle Eingang fand. In der Ausstellung symbolisiert es den Bruch und die Brutalität des Exils. 
Bohunovsky, R. - Ein Interview mit Klemens Renoldner

In einer Abschiedsgeste während seines letzten Wien-Besuchs im Jahr 1937 überließ Zweig einen Teil seiner umfangreichen und bisher wenig bekannten Autographensammlung (die handgeschriebene Werkschriften, u.a. von Franz Kafka, Thomas Mann, Hermann Hesse und Else Lasker-Schüler enthält) der Theatersammlung der Österreichischen Nationalbibliothek. Einige der wichtigsten Stücke daraus sind nun in der Ausstellung zu sehen.

Klemens Renoldner beschäftigt sich nicht erst seit 2008, als er Leiter des Stefan Zweig Centres wurde, mit dem Schriftsteller. Schon 1992 gestaltete er eine große Zweig-Ausstellung in Salzburg, die in etwas verkleinerter Form in vielen Ländern zu sehen war, so 1996 auch in São Paulo, später noch in Salvador da Bahia. Renoldner war außerdem Dramaturg am Wiener Burgtheater, Chefdramaturg der Wiener Festwochen und in der Folge an Theatern in der Schweiz und Deutschland engagiert. Daneben widmete er sich aber auch der wissenschaftlichen Arbeit, hatte mehrere Lehraufträge an den Universitäten Salzburg, Bern, Freiburg, Innsbruck und Verona inne und publiziert intensiv, v. a. zu Stefan Zweig - zuletzt erschienen 2013 seine kommentierte Ausgabe der Schachnovelle (Reclam-Verlag) und 2015 Zweigs England, das in Zusammenarbeit mit Rüdiger Görner entstanden ist (Könighausen\&Neumann). Klemens Renoldner ist außerdem Autor einer Reihe von Prosawerken, 2011 erschien der Roman über den Suizid einer 24jährigen Studentin mit dem Titel Lilys Ungeduld.

$* * *$

Ruth BohunOvsky: Die Ausstellung Wir brauchen einen ganz anderen Mut! Stefan Zweig - Abschied von Europa steht ja unter der Perspektive des Exils, sowohl inhaltlich (im Mittelpunkt stehen die Exil-Werke Die Welt von Gestern und Schachnovelle), als auch in Hinblick auf die Ausstellungsgestaltung, die um das ehemalige Luxushotel Métropole kreist (aus dem die mondäne Luxuswelt des Fin de Siècle angesichts des Nazi-Terrors weichen musste). Meine Fragen: wie kam es zur Entscheidung, bei der Ausstellung von der Exil-Perspektive auszugehen, bzw. was macht diese auch für das deutschsprachige Publikum interessant? Hat dieser Blick auch etwas mit der aktuellen politischen Situation in Europa zu tun (den Ausstellungstitel könnte man ja auch als eine Anspielung oder eine Handlungsaufforderung im Kontext der aktuellen europäischen Flüchtlingskrise verstehen)?

KLEMENS RENOLDNER: Leben und Werk Stefan Zweigs sehe ich zuerst in den 
Koordinaten der politischen Geschichte Österreichs und Europas. Und natürlich der Weltgeschichte. Und erst dann in Relation zur faszinierenden literarischen und kulturgeschichtlichen Epoche, in der er lebte. Die Habsburger Monarchie, der Erste Weltkrieg, die Demokratie der Ersten Republik Österreich, dann die Diktaturen in Deutschland und in Österreich, dann der Zweite Weltkrieg - und auch alle diese Wechsel, Übergänge und Transformationen haben Zweig geprägt und intellektuell herausgefordert, sein besonderes Autorschafts-Modell $\mathrm{zu}$ entwickeln und $\mathrm{zu}$ modifizieren. Wir sehen weiters auch die beiden Pole, zwischen die sein Werk gespannt ist: das geistige Leben Wiens und Österreichs einerseits und der Kulturkontinent Europa mit seinen Traditionen andererseits. Für ihn spielten nicht nur literarische und intellektuelle Trends aus Österreich und Deutschland eine Rolle, sondern auch aus Italien, Frankreich, England, Russland etc.

Zwei Weltkriege hat Zweig erlebt, dann Hitlers Diktatur ab 1933, mit den Auswirkungen auf die Politik in Österreich. Die Verfolgung und Ermordung der Juden durch die Nationalsozialisten machten ihn zum Emigranten. Es wird immer wieder gesagt, und mit einigem Recht, dass Zweig ein eher unpolitischer und oft ängstlicher, sehr zurückhaltender Mensch war, der sich nicht öffentlich wehren wollte, der nicht "kämpfen" wollte, der ein Opfer der Geschichte wurde, wider Willen.

Aber gleichzeitig muss man auch sehen, dass er die politischen Entwicklungen und Umwälzungen in Europa sehr genau beobachtet hat, dass er bestens informiert war und - wie man zum Beispiel im Briefwechsel mit Romain Rolland sehen kann meist sehr prononcierte Meinungen hatte. Wir erleben seine Haltung oft als sehr ambivalent. In Zweigs Exil-Jahren kann man diese Ambivalenz, Nähe oder Distanz zum realpolitischen Geschehen, unmittelbare Betroffenheit und allzu diskretes Schweigen zu Geschichte und Politik, sehr gut beobachten.

Wir wollten diesmal also keine biographische Ausstellung machen, uns hat nicht die private, intime Lebensgeschichte Zweigs interessiert, sondern seine Suche nach einer adäquaten intellektuellen Position in den Jahren des Exils. Schon 1992, zum 50. Todestag von Stefan Zweig, haben Peter Karlhuber und ich in Salzburg eine große Ausstellung über ihn gemacht, die gewissermaßen sein gesamtes Lebenswerk präsentieren sollte. Sie war überraschenderweise auch international sehr erfolgreich und ist (in einer etwas verkleinerten Version) auch in Brasilien an verschiedenen Orten gezeigt worden. Verständlich: Wir wollten uns natürlich nicht wiederholen. Und wir 
wollten eben auch eine Ausstellung zu Zweig mit besonderem Blick auf Politik und Literatur machen, und so fragten wir uns, über welche Bücher wir etwas erzählen wollen.

Wir entschieden uns für Schachnovelle und Die Welt von Gestern. Es sind zwei seiner letzten abgeschlossenen Texte, in denen Zweig direkt auf die Zerstörung seiner eigenen Lebenswelt in Österreich Bezug nimmt. Alte und Neue Welt, Katastrophe und Utopie, Europa und Exil, bürgerliche alte Welt und Neuanfang im Ungewissen - das sind die thematischen Pole, die diese beiden Bücher bestimmen. In anderen Büchern, die nach 1933 geschrieben wurden, ich meine jene über Erasmus von Rotterdam und Johannes Calvin und seinen Widersacher Castellio, wählt Zweig historische Analogien zu den Fragen von Diktatur und Toleranz - er meint sie in der Reformationsgeschichte zu finden. Dieses "Ausweichen” in die Historie war bei Exil-Schriftstellern populär und natürlich verständlich. Auch diese Bücher haben wir schließlich ergänzend hinzugefügt. Und natürlich haben wir zusätzlich auch das Buch Brasilien - Ein Land der Zukunft in die Ausstellung integriert. Brasilien spielt überhaupt eine große Rolle in der Ausstellung.

Bei der Entscheidung für Schachnovelle und Die Welt von Gestern dachten wir auch daran, dass es sich um zwei von Zweigs bekanntesten Büchern handelt, und weiters, dass wir hier die politische Parallele zur Gegenwart deutlich machen können: Zweig flüchtete von einem Land zum anderen, um Hitler und dem Krieg zu entkommen. Dieses Schicksal verbindet ihn mit tausenden Menschen heute. Wir haben in Wien an den Anfang der Ausstellung einen Text gestellt, den Zweig über das Schicksal der jüdischen Flüchtlinge geschrieben hat - und wie er das sehr konkret, sehr real beschreibt, das klingt absolut zeitgemäß. Auch in den Schulen kann man bei diesem Thema etwas über Zweig erfahren - und zugleich über aktuelle Politik ins Gespräch kommen, diese Verbindung war uns immer sehr wichtig. Zweig sollte nicht als eine Figur der Historie, als "Mann von gestern" gezeigt werden, sondern mit seiner Lebensgeschichte sind wir zugleich bei den Fragen in unserer Gegenwart. So muss man doch Literatur ansehen, dass sie etwas mit uns zu tun hat, Fragen an uns stellt.

Peter Karlhuber hatte dann die großartige Idee für die Ausstellung: Dass wir auf der einen Seite die glückliche, luxuriöse, bürgerliche Welt von Zweigs Erfolg zeigen, in der Zweig gelebt hat. Wir geben Informationen und Hinweise auf seine Position im Literaturbetrieb, seine Anerkennung in der ganzen Welt, sein faszinierendes Netzwerk 
von Künstlern und Intellektuellen. Man sieht Übersetzungen, Bilder aus der Zeit des glücklichen, unbeschwerten Reisens. Auf der anderen Seite zeigen wir, wie diese heile, gutbürgerliche Welt zerstört wird, wie aus einem Wiener Luxushotel der Ort von größter Bestialität werden kann. Das frühere Grand-Hotel "Métropole" in Wien ist ein zentraler Schauplatz der Binnenhandlung in der Schachnovelle - es repräsentiert genau diese beiden Pole unserer Ausstellung, denn es zeigt die Wende vom bürgerlichen Luxus zum faschistischen Terror. Diese Spannung ist das geheime Zentrum unserer Schau.

BOHUNOVSKY: Sie erwähnen, dass Brasilien in der aktuellen Zweig-Ausstellung eine wichtige Rolle einnimmt. Können Sie dazu etwas mehr erzählen?

RENOLDNER: 1936 reiste Stefan Zweig zum ersten Mal über den Atlantik nach Brasilien. Ein Foto, das Zweig auf dem Schiff “Alcantara” zeigt, ist das einprägsame Erkennungszeichen der Ausstellung. Eine Vitrine zeigt Dokumente zu Zweigs Arbeit an dem Brasilienbuch, eine Landkarte - die uns die "Casa Stefan Zweig" in Petrópolis zur Verfügung gestellt hat - dokumentiert alle Reisen Zweigs in Brasilien, die er für seine Recherchen angestellt hat. Weiters werden mehrere Bilder seines Aufenthaltes in Brasilien gezeigt, unter anderem auch jenes Foto mit Getúlio Vargas.

Die “Casa Stefan Zweig” und die Nationalbibliothek in Rio haben uns Manuskripte, Bilder und Dokumente zur Verfügung gestellt, die wir in Reproduktionen zeigen können. In München konnten wir zusätzlich auch den Dokumentarfilm des brasilianischen Regisseurs Sylvio Back zeigen, Stefan Zweig - Der inszenierte Tod, ein großartiger Film ${ }^{3}$, in dem Zweigs Freunde und Zeitzeugen erzählen. Man könnte noch erwähnen, dass die Schachnovelle ja in Brasilien geschrieben wurde, und dass auch mehrere Bücher aus Brasilien zu sehen sind, Erstausgaben von der Schachnovelle und der Welt von Gestern und auch ein Band der brasilianischen Werk-Ausgabe. Fotos zeigen weiters den Verleger Abrahao Koogan und seine Frau, Freunde und Bekannte in der brasilianischen Welt. Wir zeigen Zweigs Abschiedsbrief aus Petropolis vom 22. Februar 1942, die brasilianische Sterbe-Urkunde des Arztes und anderes mehr. Das wichtigste aber ist dieses Foto von 1936, Zweig im Alter von 55 Jahren, auf dem Schiff aus Europa in die "Neue Welt", das wie ein Leitbild über der Ausstellung zu denken ist.

3 Der Film kann auf YouTube gesehen werden:

https://www.youtube.com/watch?v=NmACHd8yHjs. (31/10/15)

Pandaemonium, São Paulo, v. 18, n. 26, Dez. /2015, pp. 214-235 
Bohunovsky, R. - Ein Interview mit Klemens Renoldner

BoHUNOVSKY: Wie die Millionen Flüchtlinge, die momentan nach Europa strömen, ließ Zweig also ein ganzes Leben hinter sich - und scheiterte schließlich am Versuch, sich in Brasilien ein neues aufzubauen. Dabei war Zweig nicht einer unter Millionen, sondern stets ein privilegierter Exilant. Anders als im Fall der meisten seiner Schriftstellerkollegen, die ebenfalls vor den Nazis flohen, fiel Zweigs Wahl auf Brasilien, und das war kein Zufall (wie beispielsweise bei Ulrich Becher, der stets die USA im Blick hatte und in Brasilien nur auf seine Weiterreise wartete). Angeregt durch die Lektüre der Bücher Hermann Graf KEYSERLINGS, v. a. der Südamerikanischen Meditationen, und persönliche Gespräche mit diesem polemischen Philosophen sah Zweig Südamerika als einen interessanten Gegenpol zu Europa. Schon 1928, also Jahre vor seinem ersten Brasilienbesuch, sprach er von den "neuen Impulsen" für die Welt, die von Ländern wie Brasilien oder Argentinien kommen würden. Die Idee, dass außereuropäische Länder zur "Regenerierung” der Welt beitragen könnten, prägt das Denken Keyserlings und in Folge auch jenes Stefan Zweigs, der sich als Fürsprecher Brasiliens in Europa verstand und dessen Buch Brasilien - Ein Land der Zukunft ja Ausdruck des (utopischen?) Traums der Völkerverständigung ist. Zweig kam also mit einer philosophisch-literarisch geprägten Vorstellung des Landes nach Brasilien. Hat er an diesem Bild festgehalten? Oder, gewagter formuliert: war sein Selbstmord ausschließlich eine Reaktion auf den Verlust seiner "Welt von gestern” oder vielleicht auch auf die Erkenntnis, dass die "Welt von morgen”, nämlich Brasilien, ev. nicht ganz seinen utopischen Vorstellungen im Sinne Keyserlings entsprechen könnte?

RENOLDNER: Ich sehe es nicht so eindeutig, dass Zweig sich in Brasilien ein neues Leben aufbauen wollte. Ich denke vielmehr, Brasilien war eine Option für ihn. Eine Möglichkeit unter mehreren. Die endgültige Entscheidung, in diesem Land eine neue Heimat zu finden, fehlte noch für ihn. Soweit, so glaube ich, war er noch nicht. Das hätte dann, später, danach, an einem besonderen Ort stattfinden können. Brasilien war also - so sehe ich es - nicht die Endstation für Zweig, sondern nur eine Zwischenstation des Exils auf dem Weg zu einem anderen, dem richtigen Ort, der Heimat bedeuten konnte. Aber diesen Ort gab es für Zweig nicht mehr, es hätte vermutlich doch ein Ort in Europa sein sollen, in der verlorenen "Welt von Gestern". Das aber war mitten im Krieg 1942 völlig unmöglich.

Als Zweig im Frühjahr 1940 - die Engländer hatten berechtigte Angst, dass Hitler nach der Okkupation von Frankreich auch Großbritannien militärisch besetzen 
würde - entschieden hatte, den Kontinent Europa zu verlassen, waren die USA zuerst sein Ziel. Zweig war sehr enttäuscht, er spricht in Briefen mehrmals darüber, dass er von der amerikanischen Botschaft in London nur ein Transit-Visum erhalten konnte. Durch seine guten Verbindungen mit Brasilien kam aber gleichzeitig ein unbefristetes Visum für Brasilien zustande. Und mit der Idee das Brasilien-Buch zu schreiben, das er nach seinem Aufenthalt 1936 ja schon geplant (und einige Kapitel schon geschrieben) hatte, hielt er sich dann etwa fünf Monate in Brasilien auf. Er reiste an verschiedene Orte, recherchierte und schrieb das Buch. Das zeigen wir in der Ausstellung. Von Januar bis September 1941 hielt er sich aber wieder in den USA auf, er wohnte an verschiedenen Orten, in New York, New Haven und schließlich in Ossining im Staate New York.

Zweig ist durch die Jahre seiner Emigration (ab 1934) "entwurzelt" worden, er hat sich, wie er sagte "entheimatet". Er war, wie man den Briefen entnehmen kann, nervlich sehr zerrüttet, unsicher wie und wo sein Leben weitergehen sollte und könnte, er überlegte dann abwechselnd verschiedene Exil-Länder und Städte als Aufenthaltsorte für sich und seine zweite Frau Lotte, ja er hofft zwischendurch (irrationalerweise) immer wieder vielleicht doch nach England zurückkehren zu können, wo er sich 1939 ja ein neues Haus gekauft hatte. Brasilien und Petrópolis sind für mich weitere, aber eher unsichere Zwischen-Stationen auf seinen unsicheren Irrwegen im Exil, Orte des Improvisierens und Überlegens, wie und wo das Leben dann, später, weitergehen könnte, wo man sich wieder fest und auf Dauer niederlassen könnte.

Brasilien, so sehe ich das, war — nochmals gesagt - eine von mehreren Optionen - auch dieser winzige Bungalow in Petrópolis wäre für einen längeren Aufenthalt gar nicht geeignet gewesen, das Haus war viel zu beengt. Auch wenn sie, wie sie anfangs in Briefen schrieben, heimatliche Assoziationen haben und an österreichische Landschaften (Semmering, Salzkammergut, Bad Ischl) denken, war es nicht Heimat. Lotte und Stefan Zweig waren hier sehr einsam und sehr abgeschieden, sie litten zusehends unter dieser (ja, zweifellos selbstgewählten) Einsamkeit, man konnte nur wenige Bekannte treffen, die engen Freunde, mit denen man längere Abschnitte gemeinsamen Lebens verbracht hatte, gab es hier nicht, deswegen wird die Korrespondenz mit Friderike, mit der er sich doch rund um Hausverkauf in Salzburg und um die Scheidung so heftig gestritten und gequält hatte, wieder so wichtig. Vor allem aber war in Brasilien die Sprache eine enorme Hürde: Was in England und 
Frankreich für ihn möglich war, mit Intellektuellen und Künstlern zu diskutieren und zu sprechen, das war hier nur schwer möglich. Und ganz wichtig und bitter: Zweig konnte nicht in den Bibliotheken arbeiten, weil er nicht Portugiesisch konnte. Und die konzentrierte Arbeit in Bibliotheken war für ihn, wie wir wissen, immer äußerst wichtig.

Auch die USA waren in diesem Sinne nur eine Durchreise-Station. Wir wissen, dass es in den USA verschiedene Überlegungen gab, Zweig einzuladen. Aber er wehrte ab. Er hat auch - man denke an seine Versuche, für Giuseppe Borgese oder Livinia Mazzucchetti Stellen an US-amerikanischen Universitäten zu finden - gesehen, wie schwierig die Situation selbst für exzellente Wissenschaftler war. Und zudem beteuerte er mehrere Male, dass die "angloamerikanische Sphäre" ihm nicht zusage - eine für mich etwas merkwürdige Auskunft, weil er doch sechseinhalb Jahre in Großbritannien gelebt hat.

Zweigs Brasilien-Buch versuche ich als Parallel-Buch, ja in gewissem Sinne auch als eine Art Vorstudie zur "Welt von Gestern” zu lesen. Viele Utopien, Ideen und Sehnsüchte, die er mit der österreichischen Welt verbindet, sucht er nun auf verdeckte Weise in Brasilien $\mathrm{zu}$ formulieren: Der Vielvölkerstaat Österreich und das Vielvölkerreich Brasilien weisen für ihn viele Parallelen auf. Die Utopie Brasilien ist in gewissem Sinne auch eine Version seiner Utopie von Europa. Es war eben doch ein weiteres Buch in einer Reihe von Schreib-Anlässen, wie so manche andere auch, und kein gültiges Lebensprogramm. Das heißt, so wie bei anderen Büchern hatte sich Zweig dann auch den nächsten Projekten zugewendet. Dieses Kapitel "Brasilienbuch" war für ihn abgeschlossen. Über die Resonanz des Buches ist ja viel gesagt worden, vor allem von Alberto Dines.

Mir gefällt diese Idee am besten, dass man alle Bücher Zweigs aus den Jahren des Exils (von 1934 bis 1941, von Erasmus über Castellio, Magellan, Ungeduld des Herzens, zu Brasilien, Die Welt von Gestern, Clarissa, Montaigne und Schachnovelle) als ein großes intellektuelles Projekt untersuchen und darstellen könnte. In Relation mit dem Blick zurück in die Welt Europas vor 1933 versucht er, die Ideale und Werte zu skizzieren, die ihn ein Leben lang geistig wichtig waren und ihn und seine Bücher geprägt haben: kultureller Kosmopolitismus, Humanismus, Toleranz, Anti-Diktatur, der Triumph des Besiegten, die Versöhnung der Feinde (nach dem 1. Weltkrieg), Pazifismus usw. Das wäre ein großes, natürlich höchst komplexes Projekt für die Literaturwissenschaft, in dem es auch um literarische Vorbilder, um komparatistische 
Bohunovsky, R. - Ein Interview mit Klemens Renoldner

Aspekte und um Intertextualität gehen müsste.

Viele Autoren (von Döblin bis Klaus und Heinrich Mann usw.) haben auch Bücher des Rückblicks auf Europa geschrieben. Auch hier könnte man Zweig in dem Ensemble vieler Stimmen des Exils einmal darstellen. Vielleicht würden sich so manche Vorurteile gegen Die Welt von Gestern dann auflösen.

Der Suizid von Lotte und Stefan Zweig hat - wie in jedem anderen Fall von Selbstmord - natürlich sehr viele Motive und Ursachen. Es ist für mich schwierig, dass man im Falle eines Suizids das Leben eines Menschen immer in Hinblick auf dieses Ende betrachtet. Das ist sehr problematisch. Ich denke, dass die politischen Änderungen in der Politik von Getúlio Vargas und die aktuellebn Umstände in Brasilien bzw. eine Enttäuschung über die Situation in Brasilien dabei vermutlich eine eher untergeordnete Rolle spielen. Vargas war, wenn ich es richtig verstehe, in Zweigs Augen ein sehr humaner Diktator. Ich denke, dass er sich da doch geirrt hat. Auch darüber ist gerade in Brasilien viel geschrieben worden, das muss ich nicht wiederholen.

Neben den Depressionen, die Zweig auch schon vor seinen englischen Jahren kennen gelernt hatte, war vor allem der weltumspannende Erfolg Hitlers eines der wesentlichen Motive für seine Verzweiflung. Die Erfolge der Achsenmächte und das Eintreten der USA in den Krieg, auch das bevorstehende Eintreten Brasiliens in den Krieg - alle diese Umstände, einem zweiten, immer größer werdenden Weltkrieg nicht entfliehen zu können, haben Zweig, wie wir in seinen Briefen oder auch im Vorwort zur Welt von Gestern lesen können, so sehr erschüttert, dass er aufgrund seiner eigenen psychischen Disposition ein Dagegen-Kämpfen nicht mehr für möglich hielt. Vieles weitere mag dazu kommen, seine Ideologisierung von Juvenilität, mit ihrer Kehrseite, der lebenslangen Angst vor dem Alter, die Asthma-Erkrankung seiner Frau Lotte und einiges anderes mehr. Aber die Zerstörung Europas und der unwiederbringliche Verlust der Welt, aus der er kam, die in geprägt hatte, an der er leidenschaftlich hing, die seinen Lebenssinn bestimmte, in die er aber trotz aller verschiedenen Überlegungen bei einer Neuorientierung auch ideell nicht mehr zurückkehren konnte, auch nicht im Land der Zukunft, das waren in meinen Augen die zentralen Motive, die seine letzten Lebenswochen aufs äußerste beschwert haben und der Verzweiflung eine so große Macht gaben. Er sah sich als "Mann von Gestern" - und nachdem diese Welt nicht mehr existierte, war auch sein Leben in Frage gestellt. 
Bohunovsky, R. - Ein Interview mit Klemens Renoldner

BoHUnOvsKY: Meine nächsten Fragen beziehen sich auf die Zweig-Rezeption: Wie werden seine Bücher heute gelesen? Welche seiner Bücher werden gelesen? Was macht ihn für das heutige Publikum interessant? In dem von Ihnen herausgegebenen, wunderschön gestalteten Ausstellungskatalog (eigentlich ein Lesebuch) Stefan Zweig: Abschied von Europa gibt es einen Beitrag von Erika TUNNER zur Zweig-Rezeption in Frankreich (ebd. 240-251) - wo Zweig weiterhin zu den meistgelesenen Autoren deutscher Sprache zählt. Dabei versucht Tunner die Frage "Was kann die ungetrübte Begeisterung der heutigen Leser für Stefan Zweig erklären?” zu beantworten und bietet mögliche Antworten: Zweig, ein Meister der psychologischen Novelle, "beherrscht die Kunst, Menschen in Krisensituationen zu analysieren oder an den Wendepunkten ihres Lebens zu zeigen"; seine Erzählungen seien von “atemloser Spannung” und seine Werke würden dazu beitragen, die “(Wunsch)Vorstellung” des französischen Lesers in Hinblick auf Wien zu bestätigen. Stimmen Sie dieser Einschätzung zu? Trifft sie auch für die Zweig-Rezeption im restlichen Europa und weltweit zu?

RENOLDNER: Zweig wurde in alle großen (und auch kleinere) Sprachen übersetzt, das müßte man also nach Ländern und Sprachen im einzelnen ansehen. Da gibt es sehr große Unterschiede. In einigen Ländern, in denen Zweig zu Lebzeiten sehr hohe Auflagen erzielt hatte und viel gelesen wurde, ist das Interesse an ihm heute so gut wie verschwunden. Ich denke da etwa an die USA, wo er Ende der 1930iger Jahre äußerst populär war, wo er noch in den 70iger und 80iger Jahren in der Germanistik viel über ihn geforscht und publiziert wurde, wo aber heute sein Name so gut wie vergessen ist. Es gibt einige neue Übersetzungen einzelner Bücher in kleinen Verlagen, der Film von Wes Anderson The Grand Budapest Hotel (2014) und George Prochniks biographischer Essay The impossible exile (2014) haben in den Medien für einige Wochen eine gewisse Aufmerksamkeit für Zweig erzielt, aber das ändert nichts an der Tatsache, dass er heute in Nordamerika ein unbekannter Autor ist. Das gilt auch für Russland, wo Zweig durch die von Maxim Gorki angeregte große Werk-Ausgabe von 1927 damals sehr präsent und geschätzt war. 1928 besuchte Zweig die Sowjetunion, und er hat über verschiedene russische Autoren Essays und Aufsätze geschrieben.

In China wiederum ist Zweig außerordentlich populär, es gibt mehrere Übersetzungen nebeneinander, wie das übrigens - nach dem Verfall des Urheberrechts (01/01/2013)- in vielen Ländern so ist. So gibt es, nur ein kurioses Beispiel, in französischen Buchhandlungen fünf verschiedene Übersetzungen der Schachnovelle, in 
der Türkei gibt es gar 57 verschiedene Übersetzer, die sich an den Zweig-Texten versucht haben, wie ich kürzlich erfahren konnte.

Insgesamt kann man sagen, dass sich das heutige Interesse an Stefan Zweig vor allem auf seine Novellen und Erzählungen bezieht, auch auf den Roman Ungeduld des Herzens und natürlich auf die Erinnerungen Die Welt von Gestern. Auch die großen Biographien über Marie Antoinette, Joseph Fouché und Maria Stuart muss man in diesem Zusammenhang erwähnen. Völlig vergessen aber ist der Lyriker Stefan Zweig, völlig verschwunden von den Spielplänen der Theater sind seine zehn Dramen, und auch die großen Essay-Bände zur Literatur, die zu Lebzeiten erstaunlicherweise sehr hohe Auflagen erzielten, werden nur mehr wenig zur Kenntnis genommen. In Schulen werden bis heute die sehr spannend erzählten Sternstunden der Menschheit gerne gelesen.

Aufällig ist bei Bühnenaufführungen, dass man sehr häufig Zweigs Novellen dramatisiert - die 10 Dramen aber ignoriert. Auch der Roman Ungeduld des Herzens erfreut sich gegenwärtig großer Beliebtheit bei Regisseuren des Sprechtheaters.

Frankreich ist ein außergewöhnlicher Fall: Die große Luxus-Ausgabe in der Reihe der renommierten "Pléiade" im Pariser Verlag Gallimard präsentiert in NeuÜbersetzungen auf ca. 3000 Seiten das gesamte Prosa-Werk (sämtliche Erzählungen, Ungeduld des Herzens, Sternstunden der Menschheit, Die Welt von Gestern, einschließlich der beiden fragmentarischen Romane Clarissa und Rausch der Verwandlung).

Die Renaissance Zweigs in Frankreich begann 2008 und geht auf Neuveröffentlichung von einigen Novellen zurück, die in billigen, schön gestalteten Taschenbüchern präsentiert wurden. Eine sehr clevere, nachhaltige Verlagsstrategie trifft dabei auf die große Sympathie der Franzosen für die "belle époque" Wiens, für Jugendstil, Psychoanalyse, für die Umwälzungen in bildender Kunst, bei Architektur, Musik und Literatur. Ja, es sind vor allem die Erzählungen mit erotischen Verstrickungen, die das Interesse geweckt haben, aber dann hat man auch Essays zu Politik und Literatur, ja sogar Briefe übersetzt und Zweig als politische Figur seiner Zeit, als europäischen Intellektuellen gesehen. Filme, Theaterstücke, romanhafte und wissenschaftliche Bücher zu seiner Biographie erschienen, sogar eine "Graphic Novel”. Weitere Filme und Publikationen sind in Vorbereitung. 
Bohunovsky, R. - Ein Interview mit Klemens Renoldner

$\mathrm{Ja}$, ich glaube, dass diese oft tragischen, heillosen erotischen Konflikte und der Blick in "seelische Abgründe", die faszinierende Darstellung von inneren Spannungen und Ängsten, oder wenn man so will: die vielzitierte "psychologische Meisterschaft" Zweigs das wichtigste Motiv für diesen Erfolg sind. Vielleicht sind ja auch Texte wie Angst, Brief einer Unbekannten, Brennendes Geheimnis, Amok, Verwirrung der Gefühle, Phantastische Nacht, 24 Stunden aus dem Leben einer Frau und Schachnovelle die herausragenden fiktionalen Texte dieses Schriftstellers, die im Kanon der Literatur dieser Epoche Bestand haben werden.

Zweig ist in Frankreich der am meisten gelesene fremdsprachige Autor, das ist in Italien und England - wo es in den letzten Jahren auch von sehr guten kleineren Verlage eine ganze Serie von neuen Ausgaben (mit neuen Übersetzungen!) gegeben hat - nicht in diesem Ausmaß der Fall. Interessant ist auch diese Diskrepanz: dass Zweig in der Germanistik in Deutschland und Österreich (und wohl in vielen anderen Ländern auch) doch meistens eher als "Trivialautor" abgetan wird, weil er - zugegeben - kein ästhetischer Neuerer oder avantgardistischer Revolutionär war. Und weil Hofmannsthal oder Musil den Kollegen Zweig verachtet haben, und weil diese Autoren eben gerade en mode sind, wird man auch weiterhin Zweig als "Autor sechster Güte "(Hofmannsthal) ansehen. Das hat u.a. auch mit der Ideologisierung des Romans als prestigeträchtigster Gattung zu tun, die es in anderen Ländern nicht in diesem Ausmaß gibt. Man könnte noch weitere Gründe nennen.

BOHUNOVSKY: Apropos "erotische Verstrickungen": die Zweig-Rezeption im deutschsprachigen Raum wird ja momentan von einem polemischen Buch über sein Sexualleben geprägt, Stefan Zweigs brennendes Geheimnis - eine "Intim-Biographie”, verfasst vom Wiener Jornalisten Ulrich Weinzierl. Ein Buch mit kaum neuen, aber mitich zitiere aus Ihrer Rezension - "akribischem Fleiß" und in "pointierter Weise vorgetragenen" Informationen. Inwieweit ist diese Publikation nicht nur biographisch, sondern auch kulturhistorisch, bzw. in Hinblick auf Zweigs Literatur aufschlussreich, bzw. relevant?

RENOLDNER: Die deutschprachige Zweig-Rezeption wird durch das Buch von Ulrich Weinzierl wohl kaum verändert. Zweig ist halt wieder "im Gespräch", das ist ja sehr gut, aber ich bin unsicher, ob das viele neue Leser bringt. Das Problem des Buches 
ist diese ausschließliche Fixierung auf Zweigs Sexualleben. Mir ist kein Schriftsteller der Weltliteratur bekannt, dem eine solche zweifelhafte Ehre zuteil wurde, dass ein ganzes Buch nur davon handelt. Warum es jetzt Zweig erwischt hat, das muss man Weinzierl fragen. Viele schütteln auch den Kopf, denn 1992 hat Weinzierl schon einmal eine Sammlung vieler böser und bitterer Kommentare gegen Stefan Zweig herausgegeben. Man kann sich fragen, warum jemand über einen Autor, den er im Grunde nicht schätzt, zwei Bücher schreibt. Man kann natürlich nicht umhin, hier eine Absicht zu vermuten, das Ansehen Zweigs zu beschädigen. Er ist jetzt nicht nur der massentaugliche "Trivialautor" sondern zugleich noch ein ekelhafter Perverser, der als Exhibitionist kleine Mädchen in sadistischer Motivation erschreckt und - wie Weinzierl anmerkt - auch heute in Europa straffällig wäre. Der weltberühmte Autor könnte heute wegen eines Sexualdeliktes im Gefängnis sitzen - das ist der Kern.

Wer die diversen Zweig-Biographien, biografische Forschungen, Zweigs Briefe und Tagebücher gelesen hat, der wusste seit langem von seinen Beziehungen und Affären mit verschiedenen Frauen und auch von seinen homoerotischen Neigungen. Das ist ja wahrlich nichts Neues. Und das haben auch einige Kritiker in den großen deutschen Zeitungen mit Enttäuschung angemerkt, dass der Großteil der Materie bekannt ist. So wie Weinzierl (im ersten Kapitel des Buches) die Beziehung zwischen Friderike und Stefan Zweig darstellt, so ungefähr in diesem Sinne, wenn auch nicht mit sovielen Fußnoten, habe ich in zahlreichen Vorträgen oder selbst bei Führungen in unseren Ausstellungen diese Beziehung dargestellt.

Weinzierl hat dann - im zweiten Kapitel - biographische Recherchen über einige der homosexuellen (und weniger bekannten) Freunde Zweigs geliefert, wie Erwin Rieger, Erich Ebermayer, Hans Müller-Einigen u.a. Das ist natürlich sehr verdienstvoll. Sein Hauptaugenmerk gilt aber (im dritten Kapitel) unbestreitbar einigen Tagebucheintragungen, mit deren Hilfe Weinzierl die Auftritte des jungen Autors als Exhibitionist in Wiener Parks beweisen möchte. Dieses Thema wird ja in den Biographien wiederholt als Gerücht (das Zweigs Freund Benno Geiger verbreitet hat) dargestellt. Weinzierl ist nun fest überzeugt, dass die bisherige biographische Forschung über Zweig in diesem Punkt untauglich ist, und dass er nun erstmals die ultimativen Beweise liefert. Gut also: Es stimmt wohl, dass Zweig ein exhibitionistisches Problem (und also eines mit seiner sexuellen Identität überhaupt) hatte, obwohl man zugleich dieses oder jenes Tagebuch-Zitat, das Weinzierl in seinem Sinne liest und interpretiert, 
Bohunovsky, R. - Ein Interview mit Klemens Renoldner

doch auch ganz anders lesen könnte. Die Indizienkette ist doch sehr dünn, und das ist für mich das Problem. Das wurde in der kontroversen Rezeption des Buches auch wiederholt angemerkt. Auch seine Versuche, direkte kausale Verbindungen zu biographisch-haltbaren "Auskünften” aus Lyrik und Prosa Zweigs (z. B."Phantastische Nacht”) abzuleiten, und aus einem lyrischen Ich, aus dem Verhalten von literarischer Figuren doch etwas kurzschlussartig (und auf eine germanistisch unhaltbare Weise) einen Beleg für seine biographischen Thesen abzuleiten, sie sind doch etwas verwunderlich bei einem Autor, der mit seinen Studien zu Schnitzler und Hofmannsthal doch einige Anerkennung gefunden hat, gerade auch in der Germanistik.

"Sex sells" schrieb die Neue Zürcher Zeitung, und die Äußerungen des Verlags, der sich über den kleinen Medien-Hype begreiflicherweise freut, belegen die Absicht. Aber ich bin sicher, wenn diese Welle der kleinen Erregung wieder vorüber ist, wird man auf die Desiderata der Forschung zurückkommen und sich mit den für das Werk wesentlichen Themen befassen. Das ist ja das Problem von Weinzierls Buch, dass er das literarische Werk Zweigs (sieht man von seinem Kommentar zu Verwirrung der Gefühle ab) völlig ausklammert. Und das ist auch das, was viele Literaturwissenschaftler, wie ich aus zahlreichen E-Mails von Kollegen aus aller Welt weiß, doch sehr irritiert hat. Was folgert man aus dieser Erkundung? Die Diskussion über Frauen- und MännerBilder in Zweigs fiktionalen Texten hätte doch - in Relation dazu - eine Menge Stoff geboten. So ist es eben leider eine private Intim-Biographie geworden, man vermißt die kulturgeschichtliche Weiterung. Auch Zweigs durchaus bemerkenswerte Passage "Eros Matutinus" aus der Welt von Gestern kommt in diesem Buch nur am Rande vor, dabei erzählt sie uns viel über seine Sicht der Dinge.

BoHUNOvsKY: Zurück zu den Büchern Stefan Zweigs und ihrer Rezeption: Die Welt von Gestern und Brasilien - Ein Land der Zukunft sind von Anfang auch oft kritisiert worden. Schon im Erscheinungsjahr 1941 wurde das Brasilien-Buch von Seiten (regime-)kritischer brasilianischer Jornalisten vernichtend rezensiert; Izabela KESTLER sprach 1992 von einem “Märchenbuch”. In einer Ausstellung über den Wiener Kreis (Universität Wien, 2015) war ein Exemplar der englischen Erstausgabe der Welt von Gestern zu sehen, aus dem Nachlass des Wirtschafts- und Wissenschaftstheoretikers Otto Neurath. Die ersten Seiten sind übersät mit kritischen handschriftlichen Kommentaren von Neurath (Beispiele: " a rather grotesque description of the past as an 
Bohunovsky, R. - Ein Interview mit Klemens Renoldner

ideal of peace and security. The Metternich-tradition: terrible!"; oder, als Kommentar zum Zweig-Satz "Each family hat its fixed budget": "what about the unemployed, the poor?"). Mich interessiert, wie es dazu kommt, dass auch diese Bücher, die anscheinend keiner kritischen Analyse standhalten, noch immer (gern) gelesen und neu übersetzt werden? Dienen die utopisch-nostalgisch-harmonisierenden Darstellungen der beiden Länder in diesen "Parallel-Büchern", wie Sie sie genannt haben, heute noch als Identitätsangebote? Siegt, wie Zweig im Balzac schreibt, die "Dichtung über die Geschichte”, die Fiktion über die Fakten?

RENOLDNER: Es ist sehr interessant, dass Sie auf Otto Neuraths Notizen anspielen, in dieser tollen Ausstellung über den "Wiener Kreis" in der Wiener Universität, ein neues, von Friedrich Stadler entdecktes Dokument zur Rezeptionsgeschichte. Ja, diese Art von Kritik an der Welt von Gestern hat eine sehr lange Geschichte. Die vielzitierte Kronzeugin dieser Position ist Hannah Arendt - eine jüdische Emigrantin aus Europa wie Zweig! - die nach dem Krieg eine Rezension des Buches zu einem polemischen Essay gegen Zweig gemacht hat. Sie konstatiert bei Zweigs Kommentaren zu seinem Exil eine ihr unbegreifliche bürgerlich-luxuriöse Überempfindlichkeit, und vermisst, dass er sich nicht als ein verfolgter Jude begreifen kann, sondern dass er sich als europäischer Kulturbürger geriert. Sie bemängelt auch, dass Zweig die Realpolitik der österreichischen Monarchie, die Nationalitätenkonflikte und die sozialen Probleme in Wien und Österreich nicht dargestellt hat. Diese negative Rezeptionslinie gibt es bis heute.

Ich finde durchaus, dass Die Welt von Gestern einer kritischen Analyse standhält. Es werden mit der Geste der Unzufriedenheit viele Erwartungen an dieses Buch herangetragen, aber hat man sich gefragt, was Zweig wirklich wollte? Seine Konzeption sah vielleicht doch ganz anders aus, als wir heute vermuten. Da muss man genauer sein. Manchmal kommt es einem so vor, dass man einer grünen Bank vorhält, dass sie nicht blau ist. Aber, ich sehe das immer wieder: Dieses Buch wird selbst von Zweig-Verächtern in der internationalen Germanistik sehr gerne zitiert, ja es gibt so eine Art Standard-Satz, den ich von vielen Wissenschaftlern gehört habe, und der klingt ungefähr so: "Ich schätze Zweig als Autor im Grunde nicht besonders, aber seine Autobiographie ist ein faszinierendes Buch, das ich bei meinen Forschungen/Lehrveranstaltungen immer gerne verwende”. So ungefähr.

Denn Die Welt von Gestern hat trotz der bekannten Einwände, von denen man 
auch einige teilen kann, auch sehr viele Vorzüge. Wie eben jenes Kapitel über die sexuelle Moral und Doppelmoral um 1900. Ich denke es ist eine kulturgeschichtlich bemerkenswerte Studie zu diesem Thema, und die Männer kommen hier nicht sehr gut weg. Aber auch die politischen Einschätzungen zur österreichischen Politik der 1930iger Jahre, auch das sehr eindringliche Vorwort, und natürlich die letzten Kapitel (Hitlers Erfolg, Verfolgung der Juden, Exil-Problematik etc.) enthalten doch sehr viel an politischer Schärfe und Hellsichtigkeit. Aber diese Passagen sind weniger bekannt als jene, die die Zeit vor 1914 betreffen.

Ich sehe darin weniger ein Identifikations-Angebot, und für mich steht der "utopisch-nostalgisch-harmonisierende" Aspekt (das ist auch so ein Klischee über die WvG!) nicht im Vordergrund. Es stimmt: zwei Drittel des Buches befassen sich mit der Zeit vor 1914. Aber man wird in diesem Buch keine antidemokratische Sehnsucht nach der Monarchie, nach dem alten Kaiser finden, aus der berechtigten Kritik von Arendt oder Neurath hat man auch so eine Art common sense abgeleitet, aber das ist ein Klischee. Joseph Roth war in seiner letzten Lebenszeit wirklich Monarchist, weil er hoffte, mit einer Aktion und dem Programm eines idealisierten Alt-Österreich könnte man etwas gegen den deutschen Nazi-Wahn erreichen. Zweig hat niemals so eine Position eingenommen, er fand diese Versuche, mit Otto von Habsburg eine Fraktion zu bilden, ganz unsinnig. Also: Von Nostalgie nach der guten alten heilen Welt des Habsburg-Imperiums läßt sich in der Welt von Gestern nichts finden. Das ist ein Gerücht, eine sehr große Vereinfachung, da müsste man genau Zeile für Zeile, Seite für Seite analysieren.

Man darf aber auch nicht vergessen wann das Buch (in der letzten Fassung) geschrieben wurde, nämlich im Juli 1941 in den USA. Zweig (59 Jahre alt!) wusste, was den Juden in den Konzentrationslagern der Deutschen widerfährt, er wusste, dass der Aggressions-Krieg in vollem Gange war. Österreich, Zweigs Heimatland, war von der Landkarte verschwunden, Europa, seine kulturelle, ideelle Heimat war von antidemokratischen Regierungen zerstört, ein noch viel grausamerer Krieg als der von 1914-1918 war ausgebrochen. Angesichts dieser erschütternden, äußerst verzweifelten Situation schreibt Zweig täglich - wie wir wissen - unter schweren Depressionen leidend, an diesem Buch. Und dann hält man ihm vor, er habe nicht erwähnt, wie aggressiv Österreich gegen Serbien agiert hat in den Jahren vor 1914? Und er hat nicht erwähnt wie schlecht es den böhmischen Ziegelarbeitern in Wien geht? - Der 
Bohunovsky, R. - Ein Interview mit Klemens Renoldner

Hintergrund, und die ursprüngliche Absicht zu diesem Buch sind doch ganz andere. Dass sich jemand angesichts der Auslöschung des alten Europas, der Vernichtung der jüdischen Kultur Erinnerungen an glückliche Lebensjahre vor Augen hält, das sollte man doch verstehen können.

BohunOvsKy: Die Lektüre des Buches darf also den Kontext nicht ignorieren, in dem es geschrieben wurde. Das entspricht Ihrer eingangs gemachten Bemerkung, dass Leben und Werk Zweigs v.a. im Zusammenhang mit der politischen Geschichte Österreichs und Europas interessant sind. Um diesen historischen Kontext geht es ja auch in dem 2014 in portugiesischer und englischer Sprache erschienen Buch A rede de amigos de Stefan Zweig: sua última agenda (1940-1942), bzw. A Network of Friends, Stefan Zweig, his last address book, 1940-1942, herausgegeben unter Leitung des brasilianischen ZweigBiographen Alberto Dines. Was macht ein Adressbuch so interessant, dass es der Öffentlichkeit zugänglich gemacht wird? Gibt es diese Publikation auch auf Deutsch, bzw. ist eine solche geplant?

RENOLDNER: Ja, es ist geplant, dass dieses Adressbuch - es weist bis auf zwei kleine Eintragungen Stefan Zweigs ausschließlich die Handschrift von Zweigs zweiter Ehefrau Lotte auf - auch in Deutschland veröffentlicht wird. Ich denke, es wird 2016 erscheinen. Das besondere an diesem brasilianischen Adressbuch ist, dass es einerseits fast nur die Namen von Freunden, die wie Zweig emigriert sind, aufweist, und andererseits die von zahlreichen neuen Bekannten und Institutionen in den USA und in Lateinamerika. Viele Namen, die man erwarten würde, vermisst man hier. Es ist also ein besonderes Dokument von Zweigs neuer Orientierung im Exil, sowohl in den USA als auch in Brasilien.

Vermutlich müssen wir aber annehmen, dass Zweig auch sein früher, in England angelegtes, Adressbuch, in dem sich alle europäischen Namen und Adressen befinden, bei sich geführt hat. Aber auf den ersten Eindruck liest man das in Brasilien zurückgebliebene Verzeichnis wie das Dokument eines völligen Neuanfangs - der Abschied von Europa scheint hier vollzogen.

BoHUNOvSKY: Bei seinem Abschied aus Europa hat sich Zweig ja unter anderem auch von seiner Autographen-Sammlung getrennt, die sich ja ausschließlich aus 
Bohunovsky, R. - Ein Interview mit Klemens Renoldner

Werkschriften (und nicht aus Korrespondenzen o.̈̈.) zusammensetzt. Richard BeerHoffmann, der die Übergabe 1937 an die Österreichische Nationalbibliothek in einer Wiener Tageszeitung kommentierte, meinte es ging Zweig bei seiner Sammlung um die "Erkenntnis des schöpferischen Augenblicks in der Schrift" (MüHLEGGER-HENHAPEL in: RENOLDNER 2014: 223). Welche Bedeutung hatte diese Autographensammlung im Leben und Werk Stefan Zweigs?

RENOLDNER: Die Geschichte von Stefan Zweigs Autographensammlung ist genau dokumentiert in dem Band Ich kenne den Zauber der Schrift von Oliver MatuscheK, 2005 in Wien erschienen. Hier sind auch alle Texte Zweigs abgedruckt, die seine Begeisterung für diese Originale und seine Motive für das Sammeln erklären. Dem muss man nicht viel hinzufügen. Bis 1932/33 war das Stefan Zweigs große Leidenschaft, er muss enorme Summen ausgegeben haben, um Handschriften von Goethe, Heine, Büchner usw. oder Notenblätter von Mozart, Beethoven, Schubert, Haydn usw. zu erwerben. Er studierte daran den künstlerischen Prozess, die Materialisierung eines Gedankens, den Kampf mit einem Einfall. Und er kam mit Hilfe dieser Dokumente den Personen näher, über die er schreiben wollte.

BoHUNOVSKY: Zum Abschluss eine, bzw. einige etwas konkretere Frage zum Thema Stefan Zweig und Brasilien. In seinem letzten Wohnhaus in Petrópolis ist ja der Sitz des Vereins “Casa Stefan Zweig” untergebracht, eine Gedenkstätte für Zweig und andere Exil-Künstler. Wie sieht es mit der Kooperation zwischen Brasilien und Österreich, bzw. der Casa Stefan Zweig in Rio und dem Salzburger Stefan Zweig Centre aus? Gibt es gemeinsame Publikationen, Projekte? Und: wie stehen momentan die Chancen, die Zweig-Ausstellung auch in Brasilien zu zeigen? Interesse und Bemühungen diesbezüglich gibt es ja seit einiger Zeit, hat sich schon konkret etwas ergeben?

RENOLDNER: Die Zusammenarbeit mit der "Casa Stefan Zweig" ist eine sehr erfreuliche Sache. Alberto Dines bin ich schon mehrere Male in Deutschland begegnet, zuletzt auch hier in Salzburg, Kristina Michahelles und ich telefonieren regelmäßig miteinander. Wir haben uns mehrere Male getroffen und halten uns immer auf dem Laufenden. Gemeinsam konnten wir bereits zwei Bücher realisieren: Einen Ausstellungskatalog über Giuseppe Germani, den Stefan Zweig mit einem Brief an Mussolini aus dem Gefängnis holen konnte. Arturo Larcati vom Salzburger Zweig- 
Bohunovsky, R. - Ein Interview mit Klemens Renoldner

Centre hat dafür einen wichtigen Aufsatz geschrieben. Auch die portugiesisch-englische Facsimile-Ausgabe des letzten Adressbuches von Stefan Zweig aus dem brasilianischen Nachlass haben wir gemeinsam geplant, dafür habe ich einen Text beigesteuert. Der Band kommt, wie gesagt, 2016 auch in Deutschland heraus.

Wir arbeiten natürlich in vielen Fällen zusammen, wenn es um die Beschaffung von Texten, Fotos, Filmen, Dokumenten bzw. um Reproduktionen davon geht und helfen uns gegenseitig auf eine wunderbare Weise. Wir sprechen zur Zeit mit der Universität São Paulo über eine Stefan Zweig-Konferenz in São Paulo im Frühjahr 2017 - zum 75. Todestag Stefan Zweigs - stattfinden soll, und auch da soll natürlich die Casa-Stefan-Zweig ein Mitveranstalter sein. Natürlich soll Alberto Dines der FestRedner sein. Wir sprechen auch über weitere Buch- und Ausstellungsprojekte, die wir gemeinsam realisieren wollen. Diese Verbindung ist wirklich exzellent.

Unsere Ausstellung über Zweigs Exil-Jahre, die soviel mit der letzten Lebenszeit und mit Arbeiten, die Zweig in Brasilien geschaffen hat, zu tun hat, könnte man, ja müsste man auch in Brasilien zeigen. Viele unserer Gesprächspartner in Brasilien wünschen sich das sehr. Am 23. November 2015 eröffnen wir sie in der Deutschen Nationalbibliothek in Frankfurt. Wir sind natürlich stolz, an diesem repräsentativen Ort ausstellen zu dürfen. In Frankfurt ist die Schau bis Ende März 2016 zu sehen. Danach könnte sie nach Brasilien reisen. Vor einigen Monaten sah es ja so aus, dass die Österreichische Botschaft in Brasília die Ausstellung nach Brasilien bringen möchte, und dass auch diverse Geldgeber schon gefunden seien, die den Transport des Containers über den Atlantik sowie die Kosten für Auf- und Abbau finanzieren könnten. Alle unsere Unterlagen liegen auf dem Tisch, wir warten nur auf das Signal, dass Brasilien sagt: Ja, diese Ausstellung soll bei uns gezeigt werden. Aber vielleicht klappt es ja doch noch, wenn nicht 2016, dann vielleicht 2017 ?

Bohunovsky: Das hoffen wir sehr, eine Zweig-Konferenz in São Paulo 2017 scheint ja geradezu der ideale Kontext, ganz abgesehen vom Brasilien-Bezug der Ausstellung! Ich danke herzlichst für das interessante und anregende Gespräch! 


\section{Literaturverzeichnis}

BACK, Silvio. Stefan Zweig - Der inszenierte Tod. [A morte em cena]. Dokumentarfilm. Regie; Sylvio Back, Brasilien, 1995.

BELOCH, Israel. Org. A rede de amigos de Stefan Zweig: sua última agenda (1940-1942); A network of friends. Stefan Zweig : his last address book 1940 - 1942 / Alberto DiNES, introdução ; Israel BELOCH, organização. Alberto DINES, Kristina MICHAHELLES, Israel BELOCH, pesquisa e textos. Petrópolis : Casa Stefan Zweig, 2014.

CASA STEFAn ZwEIG. http://www.casastefanzweig.org/ (01/11/2015).

GÖRNER, Rüdiger; RENOLDNER, Klemens. Zweigs England. Schriftenreihe des Stefan Zweig Centre Salzburg. Würzburg: Könighausen und Neumann, 2015.

KeSTLER, Izabela Maria Furtado. Die Exilliteratur und das Exil deutschsprachiger Schriftsteller und Publizisten in Brasilien. Frankfurt am Main: Lang, 1992.

MATUSCHEK, Oliver. Ich kenne den Zauber der Schrift. Wien: Inlibris 2005.

MÜHLEGGER-HENHAPEL. Christiane. «Etwas wunderbar Substansloses ...» Die Autographensammlung Stefan Zweigs im Wiener Theatermuseum. In: RENOLDNER, Klemens (Hg.). Stefan Zweig - Abschied von Europa. Wien, Theatermuseum und Christian Brandstätter Verlag, 2014, S. 214-238.

RENOLDNER, Klemens. Lilys Ungeduld. Wien: Folio, 2011.

RENOLDNER, Klemens (Hg.). Stefan Zweig - Abschied von Europa. Wien, Theatermuseum und Christian Brandstätter Verlag, 2014.

RENOLDNER, Klemens. Angstlust. In: Die Presse, Wien. 19. September 2015.

STEFAN ZWEIG CENTRE SALZBURG. http://www.stefan-Zweig-centre-salzburg.at/ (01/11/2015).

TUNNER, Erika, Zur jüngsten Zweig-Renaissance. Am Beispiel: Frankreich. In: RENOLDNER, Klemens (Hg.). Stefan Zweig - Abschied von Europa. Wien, Theatermuseum und Christian Brandstätter Verlag, 2014, S. 241-252.

WEINZIERL, Ulrich. Stefans Zweigs Brennendes Geheimnis. Berlin: Zsolnay 2015.

ZWEIG, Stefan. Schachnovelle. Hg. v. Klemens Renoldner. Stuttgart: Reclam, 2013.

ZWEIG, Stefan. O mundo insone e outros ensaios. Übersetzt von Kristina Michahelles. Rio de Janeiro: Zahar, 2013.

ZWEIG, Stefan. Maria Antonieta. Übersetzt von Irene Aron. Rio de Janeiro: Zahar, 2013.

ZWEIG, Stefan. Autobiografia: o mundo de ontem. Übersetzt von Kristina Michahelles. Rio de Janeiro: Zahar, 2014.

ZWEIG, Stefan. Três novelas femininas - Medo, Carta de uma desconhecida, 24 horas na vida de uma mulher. Übersetzt von Adriana Lisboa, Raquel Abi-Sâmara. Rio de Janeiro: Zahar, 2014.

ZWEIG, Stefan. Joseph Fouché - retrato de um homem político. Übersetzt von Kristina Michahelles. Rio de Janeiro: Zahar, 2015.

ZWeIG, Stefan. Novelas insólitas - Segredo ardente, Confusão de sentimentos, A coleção invisivel, Júpiter, Foi ele?, Xadrez - uma novela. Übersetzt von Maria Aparecida Barbosa, Kristina Michahelles und Murilo Jardelino. Rio de Janeiro: Zahar, 2015. 